\title{
Leidenfrost effect: Accurate drop shape modeling and refined scaling laws
}

\author{
B. Sobac,,${ }^{1, *}$ A. Rednikov, ${ }^{1}$ S. Dorbolo, ${ }^{2}$ and P. Colinet ${ }^{1, \dagger}$ \\ ${ }^{1}$ Université Libre de Bruxelles, TIPs-Fluid Physics, C.P. 165/67, av. F.D. Roosevelt 50, 1050 Brussels, Belgium \\ ${ }^{2}$ GRASP, Physics Department, Université de Liège, B-4000 Liège, Belgium
}

(Received 24 March 2014; revised manuscript received 9 July 2014; published 21 November 2014)

\begin{abstract}
We here present a simple fitting-parameter-free theory of the Leidenfrost effect (droplet levitation above a superheated plate) covering the full range of stable shapes, i.e., from small quasispherical droplets to larger puddles floating on a pocketlike vapor film. The geometry of this film is found to be in excellent quantitative agreement with the interferometric measurements of Burton et al. [Phys. Rev. Lett. 109, 074301 (2012)]. We also obtain new scalings generalizing classical ones derived by Biance et al. [Phys. Fluids 15, 1632 (2003)] as far as the effect of plate superheat is concerned and highlight the relative role of evaporation, gravity, and capillarity in the vapor film. To further substantiate these findings, a treatment of the problem by matched asymptotic expansions is also presented.
\end{abstract}

DOI: 10.1103/PhysRevE.90.053011

PACS number(s): 47.55.D-, 47.55.nb, 68.03.-g, 47.61.-k

\section{INTRODUCTION}

It is well known that a water droplet released over a very hot surface generally does not contact the plate nor boils but rather "levitates" over a thin vapor film generated by its own evaporation [1]. Although discovered in 1756 and widely studied in connection with heat transfer technologies, this so-called Leidenfrost effect is the subject of a renewed interest nowadays [2], particularly in view of new perspectives in the field of microfluidics. Indeed, as the relatively small thermal conductivity of the vapor layer slows down the phase change process, while its low viscosity confers an extreme mobility to the drop, the control and manipulation of Leidenfrost drops turns out to be possible using ratchets or other surface structures [3-6], magnetic fields [7], or electric fields [8].

Despite this activity, the fundamental understanding of the Leidenfrost effect seems to have suffered from the lack of predictive theories accounting for the nontrivial shape of the vapor film underneath the drop. Recently, Burton et al. [9] experimentally focused on this aspect using interferometry. They observed that the vapor layer, fed by evaporation, forms a concave depression in the drop interface, limited by a much thinner annular region through which the vapor escapes (see Fig. 1). For large puddlelike drops, this vapor "bubble" grows and eventually pops up as a central "chimney" due to a Rayleigh-Taylor mechanism [10,11]. Instability of large drops can also occur in the form of "star-faceted" shapes [12]. As for small droplets, they become quasispherical and turn out to take off at the very last moments of their life [13].

Here, we tackle this problem theoretically, focusing on the determination of the vapor thickness profile thanks to a lubrication-type model including a realistic description of heat transfer and evaporation. This contrasts with the work of Snoeijer et al. [11] where a different configuration was actually studied, with a uniform upward gas flow imposed through the substrate. Closer to ours as far as the modeling of evaporation is concerned is a recent work of Pomeau et al. [14], rather detailed for droplets much smaller than the capillary length but

\footnotetext{
*bsobac@ulb.ac.be

${ }^{\dagger}$ pcolinet@ulb.ac.be
}

in fact limited to the latter as the impact of hydrostatic pressure variations on the film shape is neglected. Instead, our analysis considers the crossover between small and large droplets in a unified way (in contrast with most existing theories, e.g., Refs. $[10,14])$, and turns out to yield results in excellent agreement with experiments of Burton et al. [9] as far as the vapor film geometry is concerned. In addition, new scaling laws emerge from the theory, hence leading to an improved understanding of the Leidenfrost phenomenon refining that proposed in the celebrated work of Biance et al. [10] and in the recent work of Pomeau et al. [14].

The paper is organized as follows. The problem is formulated and the essence of our modeling approach is described in Sec. II. Numerical results for the drop shapes, including the neck-pocket structure of the vapor film underneath the drop, are presented and compared with experiment in Sec. III. The scaling laws are identified from the numerical results and justified on the basis of simple considerations in Sec. IV. A matched-asymptotics analysis within the neckpocket structure is then undertaken in Sec. V, which helps to further elucidate certain physical aspects of the problem and intricacies behind the scaling laws, although the section in question is largely technical and can be omitted at first reading. Finally, our conclusions are drawn in Sec. VI.

\section{FORMULATION OF THE PROBLEM}

Let us consider an axisymmetric drop levitating above a hot surface maintained at a temperature $T_{w}^{*}$ higher than the Leidenfrost temperature $T_{L}^{*}$ (see Fig. 1). The size of the drop is measured by $R_{\max }$, as seen from above, while the vapor film thickness is $h=h(r)$. As the evaporation of Leidenfrost drops is typically long compared to thermal and viscous relaxation times, we are looking for steady shapes only (quasistationary approximation).

Two different regions are distinguished. As in Ref. [11], the upper surface is assumed to be governed by a balance between hydrostatic and capillary pressures, i.e., just as for the equilibrium shape of a sessile drop. In dimensionless form, this simply reads $\kappa+\left(z-z_{\text {top }}\right)=\kappa_{\text {top }}$, where $\kappa$ is the curvature of the drop surface (a function of the drop shape and its derivatives) and $\kappa_{\text {top }}$ (curvature at the top of the drop 


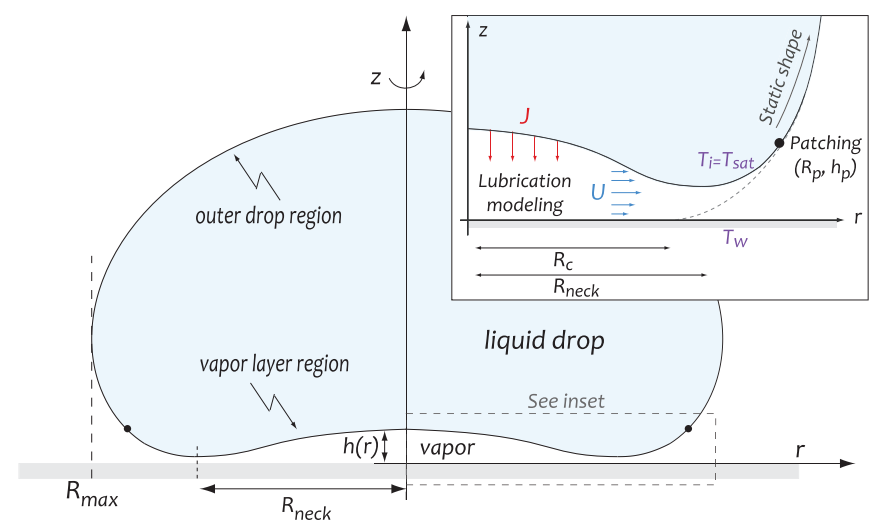

FIG. 1. (Color online) Schematic illustration of a Leidenfrost drop. The drop levitates on a vapor cushion of nonuniform thickness profile $h(r)$, composed by a pocket surrounded by an annular neck. Due to evaporation, an outward radial flow develops in the vapor layer. $R_{\max }$ is the droplet radius as seen from above, while $R_{\text {neck }}$ is the radius of the neck (at its thinnest section). The outer drop region (assumed to be an equilibrium shape) and the inner vapor layer region (described using lubrication theory including evaporation) are solved separately and numerically matched at the point of coordinates $\left(R_{p}\right.$, $h_{p}$ ). $R_{c}$ is defined from the outer drop shape as the "contact" radius of a superhydrophobic drop at equilibrium.

$\left.z=z_{\text {top }}\right)$ is a free parameter controlling its size. Note that here and throughout this paper all lengths are made dimensionless by the capillary scale $\ell_{c}^{*}=\left(\gamma^{*} / \rho_{\ell}^{*} g^{*}\right)^{1 / 2}$, where $\rho_{\ell}^{*}$ is the liquid density, $\gamma^{*}$ is the surface tension, and $g^{*}$ is the gravitational acceleration. Moreover, the presence or the absence of an asterisk is meant to distinguish between dimensional and dimensionless quantities, respectively. For a given value of the top curvature $\kappa_{\text {top }}$, numerically integrating this differential equation yields equilibrium shapes and in particular the values of $R_{\max }$ (the maximum radius) and $R_{c}$ (the radius at which the equilibrium shape has a horizontal tangent, by definition; see Fig. 1).

This "outer drop" solution is assumed to be valid up to a point located at $r=R_{p}$, between $R_{c}$ and $R_{\max }$, where nonequilibrium effects of evaporation and viscous pressure losses in the vapor flow progressively come into play. In this "vapor layer region" $0<r<R_{p}$, the thickness of the vapor film and its slopes are small enough to use the lubrication approximation. The gas itself is assumed to be composed of pure vapor (no air) that is incompressible and its properties are taken as constant. Then, ignoring possible motions inside the drop [11], the pressure in the vapor film is found from the balance of forces normal to the drop surface as $P_{v}^{*}=$ $-\left(\rho_{\ell}^{*} g^{*} h^{*}+\gamma^{*} \kappa^{*}\right)$, up to a constant. This pressure excess drives a mostly horizontal Stokes flow with volumetric flux $\vec{q}_{v}^{*}=-\frac{h^{* 3}}{12 \mu_{v}^{*}} \vec{\nabla}^{*} P_{v}^{*}$, where $\mu_{v}^{*}$ is the vapor dynamic viscosity. Note the coefficient $1 / 12$ in the mobility factor, typical of no-slip conditions imposed at both the drop surface and at the substrate.

Now, assuming that heat is only transferred by conduction across the film, the local evaporation flux at the interface is expressed as $\mathcal{J}^{*}=\mathcal{L}^{*-1} \lambda_{v}^{*}\left(T_{w}^{*}-T_{i}^{*}\right) / h^{*}$, where $\lambda_{v}^{*}$ is the vapor thermal conductivity, $T_{i}^{*}$ is the liquid-vapor interface temperature equal to the saturation temperature $T_{\text {sat }}^{*}[10]$ and $\mathcal{L}^{*}$ is the latent heat of vaporization. Finally, the classical expression of vapor mass (or volume) conservation under the lubrication hypothesis (at steady state) reads $\vec{\nabla}^{*} \cdot \vec{q}_{v}^{*}-$ $\mathcal{J}^{*} / \rho_{v}^{*}=0$, where $\rho_{v}^{*}$ is the vapor density. Combining these equations, scaling all lengths by $\ell_{c}^{*}$, and assuming the axial symmetry yields the following equation for the film thickness:

$$
\frac{1}{12} \frac{1}{r} \frac{\partial}{\partial r}\left(r h^{3} \frac{\partial}{\partial r}(h+\kappa)\right)-\frac{\mathcal{E}}{h}=0
$$

with an evaporation number $\mathcal{E}$ defined by

$$
\mathcal{E}=\frac{\lambda_{v}^{*} \mu_{v}^{*} \Delta T^{*}}{\gamma^{*} \rho_{v}^{*} \ell_{c}^{*} \mathcal{L}^{*}} .
$$

As for the curvature $\kappa$, it is here taken in a general form,

$$
\kappa=\frac{\frac{\partial^{2} h}{\partial r^{2}}+\frac{1}{r}\left[1+\left(\frac{\partial h}{\partial r}\right)^{2}\right] \frac{\partial h}{\partial r}}{\left[1+\left(\frac{\partial h}{\partial r}\right)^{2}\right]^{3 / 2}} .
$$

Apart from the size $R_{\text {max }}$ of the drop, $\mathcal{E}$ is the only parameter of the problem and depends on the fluid properties and on the superheat $\Delta T^{*}=T_{w}^{*}-T_{\text {sat }}^{*}$. While the liquid properties are taken at $T_{\text {sat }}^{*}$, the vapor properties are here evaluated at the mean temperature $\left(T_{w}^{*}+T_{\text {sat }}^{*}\right) / 2$ of the vapor film. Four boundary conditions are needed to solve Eqs. (1) and (3): symmetry conditions at $r=0$, i.e., $h^{\prime}(0)=0$ and $\kappa^{\prime}(0)=0$, while at $r=R_{p}$ the solution must match with the outer static shape of the drop, i.e., we require the continuity of $h^{\prime}(r)$ and of $\kappa(r)$. The continuity of $h(r)$ itself here merely amounts to a vertical shift of the outer shape. This problem is numerically solved using a standard second-order finite-difference method, and it is checked a posteriori that the choice of the "patching" point $R_{p}$ has an insignificant influence on the results. Note that this formulation is accurate whatever the value of $R_{\max }=$ $O(1)$, in contrast with that applied by Pomeau et al. [14], who consider either $R_{\max } \ll 1$ or $R_{\max } \gg 1$. In addition, we incorporate hydrostatic pressure variations [the term $h$ next to $\kappa$ in Eq. (1)], omitted in Ref. [14], which makes our analysis quantitative in the whole range of droplet sizes considered experimentally.

\section{NUMERICAL RESULTS AND COMPARISON WITH EXPERIMENTS}

Figure 2 shows typical shapes of Leidenfrost drops computed by our model for various $R_{\max }$. It is seen that the

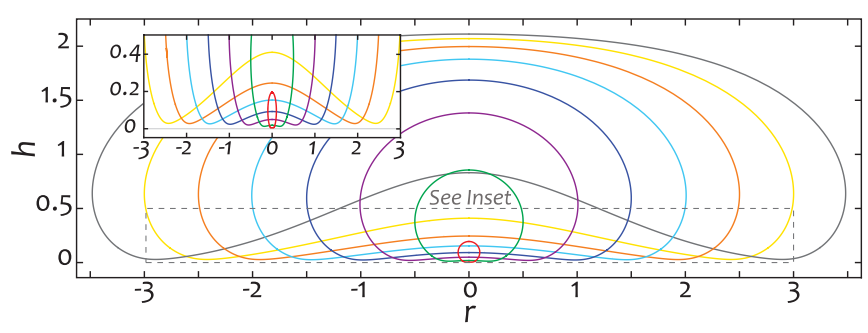

FIG. 2. (Color online) Numerically determined shapes of Leidenfrost drops for $R_{\max }=0.1,0.5,1,1.5,2,2.5,3,3.5$, and $\mathcal{E}=1.21 \times 10^{-6}\left(T_{w}^{*}=370^{\circ} \mathrm{C}\right.$ for water $)$. The lengths are scaled by the capillary length $\ell_{c}^{*}=\left(\gamma^{*} / \rho_{\ell}^{*} g^{*}\right)^{1 / 2}$ (i.e., $2.5 \mathrm{~mm}$ for water). 


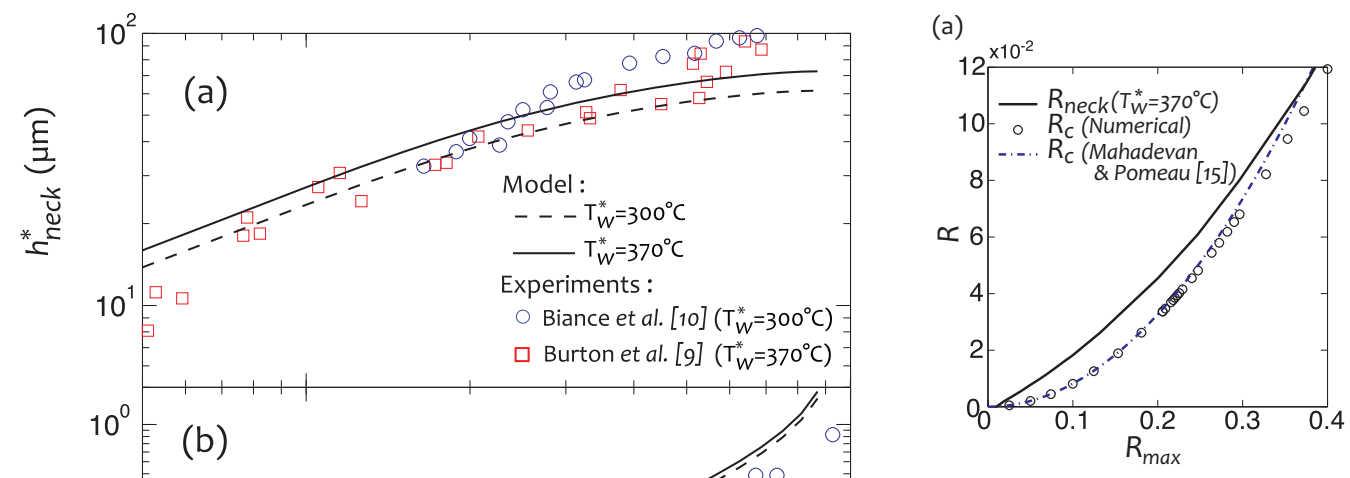

(b)

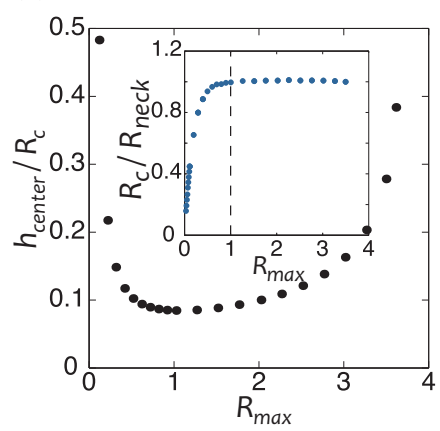

FIG. 4. (Color online) (a) Radius at the neck $R_{\text {neck }}$ and "contact" radius $R_{c}$ versus drop size $R_{\max }$ for $\mathcal{E}=1.21 \times 10^{-6}\left(T_{w}^{*}=370^{\circ} \mathrm{C}\right.$ for water). (b) Smallness parameter $h_{\text {center }} / R_{c}$ underlying the lubrication approximation versus $R_{\max }$. The inset compares $R_{\text {neck }}$ and $R_{c}$ for a larger range of sizes than in Fig. 4(a).

drop. As the "slit" thickness is here strongly nonuniform, it might be that their measurement yields an effective thickness between the actual values at the neck and at the center of the drop, i.e., always larger than $h_{\text {neck }}^{*}$ especially for large drops. The calculated thickness difference $\Delta h^{*}=h_{\text {center }}^{*}-h_{\text {neck }}^{*}$, measuring the depth of the vapor pocket, is given in Fig. 3(b) together with measurements of Burton et al. [9]. Again, the agreement is excellent, here over about 3 decades of values of $\Delta h^{*}$. Finally, Fig. 3(c) compares theoretical and experimental values of the position $R_{\text {neck }}^{*}$ of the neck, which further validates the proposed model. Note in particular the very weak influence of $T_{w}^{*}$ on both Figs. 3(b) and 3(c).

Figure 4 then highlights the subtle difference between the "contact" and neck radiuses, $R_{c}$ and $R_{\text {neck }}$ (see also Fig. 1). Indeed, this distinction is not often made in the literature, and small Leidenfrost drops $\left(R_{\max }<1\right)$ in particular are usually assumed to have a flat region at their bottom, viewed as a "contact" area of radius $R_{c}$. Moreover, the commonly used asymptotic result $R_{c}=\sqrt{2 / 3} R_{\max }^{2}$ for $R_{\max } \ll 1$ [15] is also plotted in Fig. 4(a) and seen to approximate $R_{c}$ fairly well for $R_{\text {max }}<0.35$. However, $R_{c}$ underestimates the actual extent of the apparent contact zone given by $R_{\text {neck }}$. This is clearly due to the rectification of the droplet shape by the strong evaporation occurring in the neck region. For large droplets $\left(R_{\max }>1\right)$, the inset of Fig. 4(b) shows that $R_{\text {neck }}$ and $R_{c}$ become identical, but the scaling becomes $R_{\text {neck }} \simeq R_{c} \sim R_{\text {max }}$ (puddlelike shape).

The model does not predict any "take-off" phenomenon as reported by Celestini et al. [13] for very small droplets (typically for drops with $R_{\max }^{*} \lesssim 20 \mu \mathrm{m}$ ). However, it does confirm the breakdown of the lubrication regime for $R_{\max } \rightarrow 0$, as seen from Fig. 4(b), which presents the ratio $h_{\text {center }} / R_{c}$ as a function of $R_{\max }$. This "aspect ratio" of the vapor pocket indeed needs to be small for the lubrication theory to make sense, which is seen to be the case everywhere but for $R_{\max } \rightarrow 0$ and $R_{\max } \rightarrow 4$. While the former case corresponds to spherical drops with a radius comparable to the film thickness, the latter limit coincides with the transition to a chimney (predicted at $R_{\max } \gtrsim 3.95$ [11]) rising up at the center of the puddle, indeed in agreement with experiments [10]. 


\section{SCALING LAWS FOR THE VAPOR FILM}

Importantly, new scaling laws emerge from our validated model (see Fig. 5), much more notably so for the dependence of vapor cushion characteristics on the evaporation number $\mathcal{E}$. While the neck thickness appears to scale as $h_{\text {neck }} \sim \mathcal{E}^{1 / 3}$ whatever the drop size [16], the pocket thickness grows more slowly as $h_{\text {center }} \sim \mathcal{E}^{1 / 6}$, except for very small droplets where the pocket levels with the neck and the apparent exponent gets larger (closer to $1 / 3$ ). However, at still smaller values of $\mathcal{E}$ than those represented in Fig. 5, an exponent 1/6 is recovered.

These scalings can be explained as follows. As $\mathcal{E}$ remains very small in practice, the influence of evaporation on the film shape is limited to a small region near the neck, where the film is thinnest. Denoting the radial extent of this region by $\ell_{\text {neck }}$, the typical film slope there is $h_{\text {neck }} / \ell_{\text {neck }}$, which must match with the "apparent contact angle" of the vapor pocket $h_{\text {center }} / R_{\text {neck }}$. The curvature $\kappa \sim h_{\text {neck }} / \ell_{\text {neck }}^{2}$ should remain $O(1)$, i.e., of the order of the outer drop curvature, by continuity. Moreover, from Eq. (1), the dominant balance in the neck region is found as $h_{\text {neck }}^{4} / \ell_{\text {neck }}^{4} \sim \mathcal{E} / h_{\text {neck }}$, i.e., the vapor produced in the neck is flown away through it by the local capillary pressure gradient, gravity being negligible there. Combining these estimates and considering that $R_{\text {neck }}=O(1)$, we indeed get $h_{\text {center }} \sim$ $\ell_{\text {neck }} \sim \mathcal{E}^{1 / 6}$ and $h_{\text {neck }} \sim \mathcal{E}^{1 / 3}$, as in Fig. 5. Interestingly, note that the rate of vapor production in the pocket is of the order of $\left(\mathcal{E} / h_{\text {center }}\right) \pi R_{\text {neck }}^{2} \sim \mathcal{E}^{5 / 6}$, i.e., comparable to the rate evaporated in the neck region $\left(\mathcal{E} / h_{\text {neck }}\right) 2 \pi R_{\text {neck }} \ell_{\text {neck }} \sim \mathcal{E}^{5 / 6}$. This actually contradicts the conclusions of Pomeau et al. [14], who instead find that evaporation always dominates in the vapor pocket region, where it also influences the film shape [while in our case it is an equilibrium shape governed by

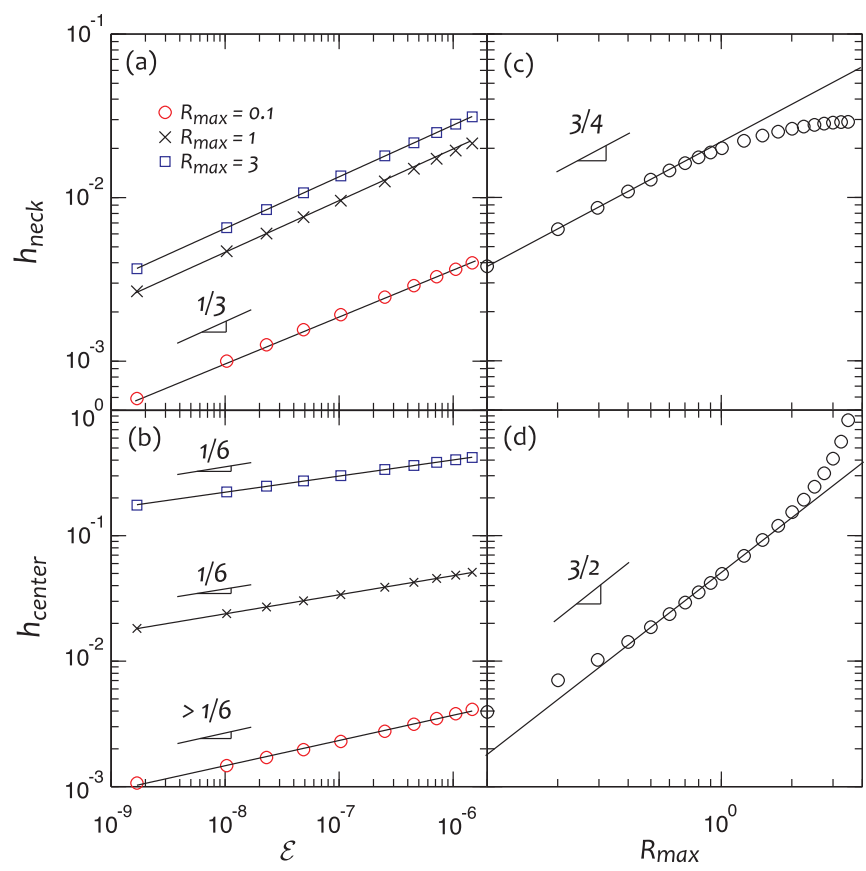

FIG. 5. (Color online) Dimensionless vapor film thicknesses (a) at the neck and (b) at the center as a function of the evaporation number for various drop sizes. [(c) and (d)] The same thicknesses versus the drop size for an evaporation number $\mathcal{E}=1.21 \times 10^{-6}$. gravity and capillarity only, just as the top of the droplet, for $h_{\text {center }}^{4} / R_{\text {neck }}^{4} \sim \mathcal{E}^{2 / 3} \gg \mathcal{E} / h_{\text {center }} \sim \mathcal{E}^{5 / 6}$ in Eq. (1)]. These important conceptual differences are associated with the slightly different scaling exponents proposed by these authors ( $1 / 3$ for $h_{\text {neck }}$ but $1 / 5$ for $h_{\text {center }}$ instead of $\left.1 / 6\right)$. Based on the numerical validation of our scalings (see Fig. 5) and on a detailed matched asymptotic analysis (see Sec. V), we believe our physical picture of the vapor film to be the correct one, however. Note in addition that these scalings actually appear to be logarithmically modulated, even though this cannot be discerned for the small values of $\mathcal{E}$ encountered in practice (see Sec. V).

As for the dependence on size, it is more delicate to construct scaling arguments valid in an appreciable range. Yet, from Fig. 5 it appears that $h_{\text {neck }}$ grows less rapidly than $R_{\max }^{3 / 4}$ while $h_{\text {center }}$ grows faster than $R_{\max }^{3 / 2}$ apart at very small sizes. Maybe expectedly (given their modeling based on a flat vapor film), the exponents $5 / 4$ and $4 / 3$ found by Biance et al. [10] for small drops lie in between the extreme ones predicted here for $h_{\text {neck }}$ and $h_{\text {center }}$.

The next section is aimed at further clarifying the findings such as the pocket-neck structure of the vapor film underneath the droplets and the scaling laws by means of matched asymptotic expansions (MAE) based on the evaporation number being typically very small in practice. Following this largely technical section, we proceed to summarizing our conclusions.

\section{MATCHED ASYMPTOTIC EXPANSIONS IN THE LIMIT $\mathcal{E} \rightarrow \mathbf{0}$}

\section{A. Preliminary remarks}

The values of the evaporation number $\mathcal{E}$, defined in Eq. (2) and entering the dimensionless formulation through the vapor film equation (1) are typically very small. For instance, as estimated in Sec. III, we have $\mathcal{E}=1.21 \times 10^{-6}$ in the case of water at $1 \mathrm{~atm}$ and a substrate temperature $T_{w}^{*}=370^{\circ} \mathrm{C}$. This suggests the utility of carrying out an asymptotic analysis in the limit $\mathcal{E} \rightarrow 0$ while at the same time considering $R_{\max }=O(1)$. Note, though, as it can already be anticipated based on the $\mathcal{E}$ scalings established in Sec. IV, the real smallness parameter of the problem is actually $\mathcal{E}^{1 / 6}$ and hence not that small $\left(\mathcal{E}^{1 / 6} \sim\right.$ 0.1 ). Nonetheless, the asymptotic analysis is still deemed of interest from the conceptual point of view, as it would permit us to further clarify the nature of the scalings and of the vapor film structure underneath Leidenfrost droplets.

A typical arrangement for an asymptotic analysis is that the structure of the solution is first reasonably guessed and then confirmed by the possibility of constructing a solution (typically its leading order in terms of the smallness parameter or a few leading orders) in the framework of this structure. In principle, a trial-and-error approach can be invoked should there be several reasonable guesses. In the present problem, however, such a guessing part is essentially spared by what we have already learned from the numerical solution in Sec. III and from a related problem considered in Ref. [11]. Namely we expect the existence of two regions in the vapor film underneath the droplet: a (relatively thick) pocket and a (narrow) neck regions, the latter in a small vicinity of 
$r=R_{c}$ (recall that $R_{c}=R_{c}^{*} / l_{c}^{*}$ is the contact radius of the corresponding equilibrium superhydrophobic droplet, i.e., the one whose upper part coincides to leading order with our Leidenfrost droplet).

According to this scheme, the leading-order pocket solution $h=h(r)$ is expected to vanish at a finite $r$ (actually at $r=R_{c}$ to the same leading order). Obviously, this is actually impossible as leading to an infinite evaporation rate at this point in the framework of our formulation, cf. the last term on the left-hand side of Eq. (1). Therefore, the trend of $h(r)$ going to zero at $r=R_{c}$ must be corrected in a small region near $r=R_{c}$, which is what constitutes the neck region. Clearly, all this implies $h_{\text {center }} \gg h_{\text {neck }}$, a result expected to be attained in the limit $\mathcal{E} \rightarrow 0, R_{\max }=O(1)$. How well this works for small but finite values of $\mathcal{E}$ encountered in practice $\left(\mathcal{E}^{1 / 6} \sim 0.1\right)$ can already be anticipated by inspecting the results presented in Figs. 2 and 3: While this seems to be alright for $R_{\max } \sim 3$, this is very much questionable for $R_{\max } \sim 1$ and smaller.

Two regions being present in the vapor-film problem, we shall here resort to the method of matched asymptotic expansions: The solutions are represented separately in each region, with the results being matched.

\section{B. First consideration in the neck region}

We first turn to the neck region. Working in a small vicinity of $r=R_{c}$, we introduce a new coordinate,

$$
x=R_{c}-r .
$$

Let $\delta \ll 1$ be the small parameter describing the longitudinal extent of the neck, i.e., $x=O(\delta)$ in the neck region. How $\delta$ is related to $\mathcal{E}$ will be established later. At one of the edges of the neck region, formally corresponding to $x / \delta \rightarrow-\infty$, the film is expected to rejoin (match) to leading order the unperturbed equilibrium shape of a sessile droplet, hence $d^{2} h / d x^{2} \rightarrow \kappa_{c}$. Here $\kappa_{c}$ is the curvature at the contact line of the equilibrium superhydrophobic sessile droplet with a contact radius $R_{c}$. As $\kappa_{c}=O(1)$, we expect $d^{2} h / d x^{2}=O(1)$ in the neck region. In view of $x=O(\delta)$, it is then clear that $h=O\left(\delta^{2}\right)$. Accordingly, we define the variables

$$
\tilde{x}=x \kappa_{c} / \delta, \quad \tilde{h}=h \kappa_{c} / \delta^{2}
$$

in the neck region such that $\tilde{x}=O(1)$ and $\tilde{h}=O(1)$ therein. The condition of matching with the equilibrium shape then becomes

$$
d^{2} \tilde{h} / d \tilde{x}^{2} \rightarrow 1 \text { as } \tilde{x} \rightarrow-\infty .
$$

Rendering Eq. (1) in terms of $\tilde{x}$ and $\tilde{h}$ and aiming at retaining only possible leading-order contributions, one obtains the equation

$$
\frac{1}{12} \frac{d}{d \tilde{x}} \tilde{h}^{3} \frac{d^{3} \tilde{h}}{d \tilde{x}^{3}}-\frac{\mathcal{E} \kappa_{c}}{\delta^{6}} \frac{1}{\tilde{h}}=0 .
$$

The factors that dropped out in Eq. (7) as compared to Eq. (1) are the hydrostatic pressure (negligible in the neck against the Laplace pressure) and the axial geometry (the neck extent being much smaller than $R_{c}$, we are just left with a planar geometry).

In view of Eq. (7), one may be tempted to relate the small parameter of the neck $\delta$ to $\mathcal{E}$ as $\delta=\mathcal{E}^{1 / 6}$ (which might be worth defining rather like $\delta=\left(\mathcal{E}_{\kappa_{c}}\right)^{1 / 6}$ or even like $\delta=\left(12 \mathcal{E} \kappa_{c}\right)^{1 / 6}$, a different order-unity factor not changing the asymptotic essence here). This actually nicely reproduces the neck-related scaling pointed out in Sec. IV and $h_{\text {neck }}=O\left(\mathcal{E}^{1 / 3}\right)$ in particular. Within a matched-asymptotics scheme, this same scaling has already been claimed by Pomeau et al. [14] (translated into our notations). However, as we shall see shortly, it proves in fact to be slightly more subtle than that, the key here being the influence on the neck region of what is going on in the adjacent vapor pocket. Furthermore, the boundary condition at the other edge of the neck, as $\tilde{x} \rightarrow+\infty$, which is essentially a matching condition between the neck and the pocket, is also naturally dependent on how the pocket is. We explore three possible pocket scenarios in the following few subsections.

\section{Pocket scenario I (not further pursued)}

A first possibility that comes to mind for the opposite-edge $(\tilde{x} \rightarrow+\infty)$ boundary condition in the neck region is just the same kind of condition we had in Eq. (6) as $\tilde{x} \rightarrow-\infty$. Namely $\tilde{h} \sim \frac{1}{2} \kappa_{b} \tilde{x}^{2}$ as $\tilde{x} \rightarrow+\infty$, where $\kappa_{b}=O(1)$ (to be determined) now corresponds to the near-contact-line curvature of the internal bubble forming the pocket. Evidently, with this asymptotics, the definitions (4) and (5), and the fact that by definition $r=O(1)$ in the pocket, the scaling for $h$ therein must be $h=O(1)$, meaning that the pocket is generally as thick as the external equilibrium shape of the droplet. This picture clearly does not correspond to the solution obtained in Sec. III. By analogy with the situation considered in Ref. [11], we interpret this as unstable pocket shapes associated with the chimney instability and no further pursue this path here. The following pocket scenario is also a counterpart of the one considered for a related problem in Ref. [11].

\section{Pocket scenario II (adopted here)}

Another possibility compatible with Eq. (7) is

$$
\tilde{h} \sim \vartheta \tilde{x} \quad \text { as } \quad \tilde{x} \rightarrow+\infty,
$$

where $\vartheta=O(1)$ (to be determined) can be interpreted as a (rescaled) apparent contact angle of the vapor pocket, the original (nonrescaled) one being

$$
\theta=\delta \vartheta
$$

on account of Eq. (5). The angle (9) is small, and thus so must be the vapor-pocket thickness in this scenario. Indeed, with (8) and on account of Eqs. (4) and (5), the scaling in the pocket region $r=O(1)$ is found to be $h=O(\delta)$. With the earlier mentioned naive approach according to which $\delta=$ $\mathcal{E}^{1 / 6}$ in order to render $O(1)$ the coefficient in Eq. (7), we thereby recover the scaling $h_{\text {center }}=O\left(\mathcal{E}^{1 / 6}\right)$ pointed out in Sec. IV for not-so-small droplets (albeit, as we have already mentioned, it will eventually prove to be slightly more subtle in the framework of the asymptotic analysis). With $r=O(1)$, $h=O(\delta)$, and $\delta$ sufficiently close to $O\left(\mathcal{E}^{1 / 6}\right)$, one can see, e.g., using Eq. (1), that the evaporation term is negligible to leading order in the vapor pocket, whose shape is then determined just by gravity and capillarity as for the external equilibrium shape of the droplet. Defining

$$
\bar{h}=h / \delta,
$$


such that $\bar{h}=O(1)$ in the pocket, Eq. (1) yields, to leading order,

$$
\frac{d^{2} \bar{h}}{d r^{2}}+\frac{1}{r} \frac{d \bar{h}}{d r}+\bar{h}=\text { const. }
$$

Its solution, smooth at the symmetry axis and satisfying the boundary condition (matching with the neck region)

$$
\bar{h}=0, \quad d \bar{h} / d r=-\vartheta \quad \text { at } \quad r=R_{c},
$$

is

$$
\bar{h}=\vartheta \frac{J_{0}(r)-J_{0}\left(R_{c}\right)}{J_{1}\left(R_{c}\right)} \quad\left(0 \leqslant r \leqslant R_{c}\right),
$$

where $J_{0}$ and $J_{1}$ are the Bessel functions of the first kind. We observe that this solution, similar to the ones pointed out in Ref. [11], can be valid only for $R_{c}<3.83$ (the number corresponding to the first root of $J_{1}$ ). Furthermore, we must require (3.83- $\left.R_{c}\right) \gg \delta$ for (12) to still represent a thin film, in which approximation the results have been derived, and this is what will be assumed hereafter. Pocket thickening for $R_{c}$ close to 3.83 is interpreted as a precursor of the chimney instability of a Leidenfrost droplet. Note that it is apparently for $\left(3.83-R_{c}\right)=O(\delta)$ that the (supposedly stable) thin-film regimes that we consider here mingle with the unstable thickvapor-pocket regimes touched on in the previous subsection.

Before proceeding further with the matching between the pocket and the neck regions, we consider yet another possible vapor-pocket configuration, ascertained by Pomeau et al. [14], but which we believe is not in fact appropriate for our problem.

\section{E. Pocket scenario III (discarded)}

Equation (7) also admits one other asymptotic behavior worth considering. To this purpose, it is no longer necessary to keep a distinction between $\delta$ and $\mathcal{E}^{1 / 6}$. More concretely, we choose $\delta=\left(\mathcal{E} \kappa_{c}\right)^{1 / 6}$, rendering Eq. (7) in the form

$$
\frac{1}{12} \frac{d}{d \tilde{x}} \tilde{h}^{3} \frac{d^{3} \tilde{h}}{d \tilde{x}^{3}}-\frac{1}{\tilde{h}}=0 .
$$

There is an exact solution to this equation, $\tilde{h}=\left(5^{4} / 2\right)^{1 / 5} \tilde{x}^{4 / 5}$, and one may suppose that this is what yields the intermediate asymptotics between the neck and the pocket, i.e.,

$$
\tilde{h} \sim\left(\frac{5^{4}}{2}\right)^{1 / 5} \tilde{x}^{4 / 5} \quad \text { as } \tilde{x} \rightarrow+\infty,
$$

instead of Eq. (8). Nonetheless, one can immediately cast doubt on the premise (13). Indeed, the dimensionless and appropriately rescaled volume flux through a vertical cross section of the vapor film, taken per unit length along the circumference of the neck, is given by $\tilde{q}_{v}=\frac{1}{12} \tilde{h}^{3} d^{3} \tilde{h} / d \tilde{x}^{3}$ within our lubrication model. With (13), one obtains $\tilde{q}_{v}>0$. This implies a flux in the positive direction of $\tilde{x}$, i.e., towards the pocket. Nonetheless, physically, one would expect exactly the opposite, namely what is evaporated in the pocket breaking outside through the neck.

As far as the associated pocket scaling is concerned, Eq. (13), on account of (4) and (5), implies in the region $r=O(1)$ that $h=O\left(\delta^{6 / 5}\right)=O\left(\mathcal{E}^{1 / 5}\right)$; in particular, $h_{\text {center }}=$ $O\left(\mathcal{E}^{1 / 5}\right)$. If so, introducing a new variable,

$$
\bar{h}=h / \mathcal{E}^{1 / 5},
$$

such that $\bar{h}=O(1)$, one finds all the terms of Eq. (1), here also including the evaporation term, to become of the same order of magnitude:

$$
\frac{1}{12} \frac{1}{r} \frac{d}{d r}\left[r \bar{h}^{3} \frac{d}{d r}\left(\frac{d^{2} \bar{h}}{d r^{2}}+\frac{1}{r} \frac{d \bar{h}}{d r}+\bar{h}\right)\right]-\frac{1}{\bar{h}}=0,
$$

which differs remarkably from the arrangement considered in the previous subsection. The earlier discussed strange consequences of Eq. (13) for the flux find in fact another manifestation in the framework of Eq. (14). Indeed, in this framework, the dimensionless rescaled flux is given by [cf. also the text before Eq. (1)] $\bar{q}_{v}=\frac{1}{12} \bar{h}^{3} \frac{d}{d r}\left(\frac{d^{2} \bar{h}}{d r^{2}}+\frac{1}{r} \frac{d \bar{h}}{d r}+\bar{h}\right)$, and Eq. (14) can be represented as

$$
\frac{1}{r} \frac{d}{d r}\left(r \bar{q}_{v}\right)-\frac{1}{\bar{h}}=0
$$

Multiplying Eq. (15) by $r$ and integrating by $r$ from 0 to $R_{c}$, one obtains

$$
\left.R_{c} \bar{q}_{v}\right|_{r \rightarrow R_{c}}-\left.\left(r \bar{q}_{v}\right)\right|_{r \rightarrow 0}=\int_{0}^{R_{c}} \frac{r}{\bar{h}} d r .
$$

In view of (13), $\bar{h} \propto\left(R_{c}-r\right)^{4 / 5}$ as $r \rightarrow R_{c}$. Consequently, $\left.\bar{q}_{v}\right|_{r \rightarrow R_{c}}=0$. Furthermore, the integral on the right-hand side of (16) converges and is obviously positive. Thus, in accordance with Eq. (16), the quantity $\left.\left(r \bar{q}_{v}\right)\right|_{r \rightarrow 0}$ must be finite and negative, which amounts to a sink at the axis. In other words, we have obtained that the vapor produced in the pocket must be consumed in an axis sink rather than getting out through the neck, which is obviously meaningless. Therefore, we discard the present pocket scenario, which was claimed (mistakenly, in our opinion) in Ref. [14].

\section{F. Neck-pocket matching conditions (continuation)}

Hereafter we adopt the pocket scenario II. One can see both physically and mathematically that the formulation given by (6)-(8) (with $\vartheta$ to be determined) is not yet sufficient for the determination of the neck solution. Physically, we expect the neck profile to be affected by the vapor flow from the pocket towards the exterior (meaning the vapor produced by evaporation in the pocket itself), whereas this factor is seemingly not yet accounted for in the formulation.

To make this point mathematically, we consider further terms of the coordinate expansion behind (8) for a solution of (7). We obtain

$$
\begin{gathered}
\tilde{h} \sim \vartheta \tilde{x}+\frac{3 \mathcal{E} \kappa_{c}}{\delta^{6} \vartheta^{4}} \ln ^{2} \tilde{x}+B \ln \tilde{x}+C+O\left(\frac{\ln ^{3} \tilde{x}}{\tilde{x}}\right) \\
\text { as } \tilde{x} \rightarrow+\infty,
\end{gathered}
$$

where $\vartheta, B$, and $C$ are free coefficients to be determined in the framework of our boundary-value problem. Likewise, we carry out a similar coordinate expansion at the opposite end of the neck region:

$$
\tilde{h} \sim \frac{1}{2} K\left(\tilde{x}-\tilde{x}_{0}\right)^{2}+H+\frac{Q}{\tilde{x}^{3}}+O\left(\frac{1}{\tilde{x}^{4}}\right)
$$$$
\text { as } \tilde{x} \rightarrow-\infty \text {, }
$$ 
here with

$$
K=1, \quad \tilde{x}_{0}=0,
$$

on account, respectively, of (6) and of choosing the reference along $x$ to match with the equilibrium droplet shape whose superhydrophobic contact radius is exactly equal to $R_{c}$ [cf. (4)]. As for $H$ and $Q$, they are free coefficients to be determined. Note that $H$ has a meaning of a (rescaled) elevation of the equilibrium part of the Leidenfrost droplet over the corresponding superhydrophobic droplet due to the presence of a vapor film underneath. Now if we look at our boundaryvalue problem as a Cauchy one starting from $\tilde{x} \rightarrow+\infty$ and advancing to $\tilde{x} \rightarrow-\infty$, we have three free coefficients in (17) while only two conditions (19) to satisfy in (18). Thus, the boundary-value problem is indeed yet underdetermined.

On the other hand, the coefficients in (17) can indeed be related to the flux coming from the pocket, the key one here being $B$. Consider the flux quantity

$$
\tilde{q}_{v}=\frac{1}{12} \tilde{h}^{3} \frac{d^{3} \tilde{h}}{d \tilde{x}^{3}},
$$

already appropriately termed in the previous subsection. Using (17) in (20), we obtain the following coordinate expansion towards the pocket region:

$$
\begin{gathered}
\tilde{q}_{v} \sim \frac{\mathcal{E} \kappa_{c}}{\delta^{6} \vartheta}\left(\ln \tilde{x}-\frac{3}{2}\right)+\frac{1}{6} \vartheta^{3} B+O\left(\frac{\ln ^{2} \tilde{x}}{\tilde{x}}\right) \\
\text { as } \tilde{x} \rightarrow+\infty,
\end{gathered}
$$

which confirms what was just outlined.

Now, to complete the formulation of the matching conditions, one can calculate the evaporation flux over the pocket region and to equate it (in an appropriately rescaled way) to (21). This can be viewed as the sought additional relation between the coefficients of (17). Alternatively, one could in principle do it by means of a direct matching of (17) with the solution for $h$ in the pocket region. However, comparing (17) with (12), we see that the logarithmic terms in (17) cannot in fact match with the leading-order pocket profile (12) shaped just by gravity and capillarity, for they must rather match with the first, evaporation-induced, correction to (12). On the other hand, proceeding in terms of the fluxes will prove to be more straightforward and spare the necessity of calculating the correction to (12). In the following subsection, we calculate the flux in the pocket. Then, we finalize the neck formulation as discussed in this paragraph.

\section{G. Evaporation flux over the pocket}

In terms of $\bar{h}$, defined in (10), Eq. (1) can be rewritten as (no approximation yet made)

$$
\frac{1}{r} \frac{d}{d r}\left(r \bar{q}_{v}\right)-\frac{\mathcal{E}}{\delta^{5} \bar{h}}=0
$$

with the flux

$$
\bar{q}_{v}=\frac{1}{12} \bar{h}^{3} \frac{d}{d r}\left(\frac{d^{2} \bar{h}}{d r^{2}}+\frac{1}{r} \frac{d \bar{h}}{d r}+\bar{h}\right) .
$$

Assuming that $\delta$ is sufficiently close to $O\left(\mathcal{E}^{1 / 6}\right)$, the last, evaporation, term on the left-hand side is negligible to leading

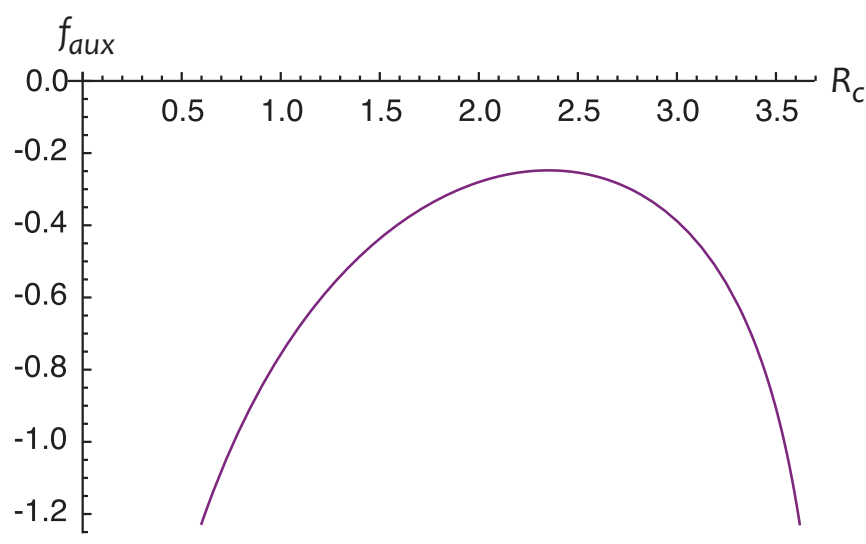

FIG. 6. (Color online) Graph of the auxiliary function defined in Eq. (26).

order. Then the pocket shape is just an equilibrium one determined by gravity and capillarity. It satisfies the equation $\bar{q}_{v}=0$, equivalent to (11) on account of no singularity at the axis, and the solution is given by (12). Now one can calculate a nontrivial result for $\bar{q}_{v}$ by using (12) in the evaporation term of Eq. (22). We obtain

$$
\bar{q}_{v}=\frac{\mathcal{E}}{\delta^{5} \vartheta} \frac{1}{r} \int_{0}^{r} \frac{J_{1}\left(R_{c}\right) r^{\prime}}{J_{0}\left(r^{\prime}\right)-J_{0}\left(R_{c}\right)} d r^{\prime}
$$

We are first concerned with the value of (24) as $r \rightarrow R_{c}$, which not only yields a quantity of interest such as the (dimensionless rescaled) evaporation flux over the entire pocket but also serves to finalize the matching with the neck region. We note, however, that (24) diverges logarithmically as $r \rightarrow R_{c}$. We deduce for it the following asymptotic behavior:

$$
\bar{q}_{v} \sim \frac{\mathcal{E}}{\delta^{5} \vartheta}\left[f_{\text {aux }}\left(R_{c}\right)-\ln \left(R_{c}-r\right)\right] \quad \text { as } r \rightarrow R_{c},
$$

where the auxiliary function

$f_{\text {aux }}\left(R_{c}\right)=\frac{1}{R_{c}} \int_{0}^{R_{c}}\left[\frac{J_{1}\left(R_{c}\right) r}{J_{0}(r)-J_{0}\left(R_{c}\right)}-\frac{R_{c}}{R_{c}-r}\right] d r+\ln R_{c}$

is evaluated numerically for each $R_{c}$. Its plot is shown in Fig. 6 . Equation (25) with (26) is the sought result of the present subsection.

\section{H. Neck-pocket matching conditions (finalization)}

Changing in (23) from the barred to tilded variables [cf. Eqs. (4), (5) and (10)] and comparing this to (20), we find that there must be the following correspondence between $\tilde{q}_{v}$ and $\bar{q}_{v}$ :

$$
\left.\tilde{q}_{v}\right|_{\tilde{x} \rightarrow+\infty} \sim-\left.\frac{\kappa_{c}}{\delta} \bar{q}_{v}\right|_{r \rightarrow R_{c}} .
$$

Using (25) in here and expressing $\left(R_{c}-r\right)$ through $\tilde{x}$ by means of (4) and (5), we obtain

$$
\tilde{q}_{v} \sim-\frac{\mathcal{E} \kappa_{c}}{\delta^{6} \vartheta}\left[f_{\text {aux }}\left(R_{c}\right)+\ln \kappa_{c}-\ln \delta-\ln \tilde{x}\right] \quad \text { as } \tilde{x} \rightarrow+\infty .
$$


We observe that the results (21), developed from the side of the neck, and (27), developed from the side of the pocket, conform to one another. The terms $\propto \ln \tilde{x}$ match automatically, whereas the constant terms yield the sought additional relation between the coefficients of (17). This implies a possibility of successful matching. Thus, the problem in the neck region is now fully determined. Note also that, on account of (20), the matching condition in question can be rewritten in the form

$$
\begin{gathered}
\tilde{h}^{3} \frac{d^{3} \tilde{h}}{d \tilde{x}^{3}} \sim-\frac{12 \mathcal{E} \kappa_{c}}{\delta^{6} \vartheta}\left[f_{\text {aux }}\left(R_{c}\right)+\ln \kappa_{c}-\ln \delta-\ln \tilde{x}\right] \\
\text { as } \tilde{x} \rightarrow+\infty,
\end{gathered}
$$

which makes no explicit reference to $\tilde{q}_{v}$ or to the coordinate expansion (17).

\section{Logarithmic modulation of power laws}

What immediately draws attention in Eqs. (27) and (28) is the presence of logarithmically large terms (logarithms of the smallness parameter $\delta$ ), which only becomes apparent after this final touch to the neck formulation, accounting for the evaporation flux from the pocket. There are consequences thereof on the asymptotic picture of the problem in the limit $\mathcal{E} \rightarrow 0$ in question.

First, the relationship between the small parameters $\mathcal{E}$ and $\delta$ is not actually given, as one could naively assume based on Eq. (7), by $\mathcal{E} / \delta^{6}=O(1)$ but rather by $\mathcal{E} \ln \delta^{-1} / \delta^{6}=O(1)$, as dictated by the dominant contribution in (27) or (28). Thus, the earlier established scaling laws $h_{\text {neck }}=O\left(\delta^{2}\right)$ and $h_{\text {center }}=$ $O(\delta)$ in particular do not in fact correspond exactly to the power laws $h_{\text {neck }}=O\left(\mathcal{E}^{1 / 3}\right)$ and $h_{\text {center }}=\mathcal{E}^{1 / 6}$ but are actually logarithmically modulated. That is, this is how it is in the asymptotic limit $\mathcal{E} \rightarrow 0$. Practically though, for small but finite $\mathcal{E}$, we do not observe any such modulation (see Sec. IV), which is not very surprising given the logarithm properties. But then again, it should be noted that, in principle, even the power laws pertinent to the asymptotic limit are not a priori guaranteed at finite $\mathcal{E}$. However, we do observe them in Sec. IV for not-so-small droplets (not-so-small $R_{c}$ and $R_{\max }$ ).

On the other hand, the "naive" scaling $\mathcal{E}^{5 / 6}$ established in Sec. IV for the global evaporation rate in the pocket and in the neck now obviously modifies to $\mathcal{E} / \delta$. However, as manifested, e.g., in Eqs. (27) or (28), this must yet be multiplied by $\ln \delta^{-1}$, which can be considered as a vestige of a resolved divergence in an intermediate zone between the pocket and the neck. Whether to attribute such an intermediate-zone contribution to the pocket or to the neck is a matter of an arbitrary convention. Here we shall divide it between them, thus keeping unmodified the conclusion that the global evaporation rate is of the same order in the pocket and in the neck, the scaling for which is now given by $\mathcal{E} \ln \delta^{-1} / \delta=O\left(\delta^{5}\right)$. For practically small values of $\mathcal{E}$, there must be no way to discern this from $\mathcal{E}^{5 / 6}$.

Let us also mention the following. If one relies on the pocket scenario III (which, as already mentioned, is deemed inappropriate here), the difference with the pocket scenario II (presently implied) evidently goes in the pocket as far as the power laws themselves. For instance, $h_{\text {center }}=O\left(\mathcal{E}^{1 / 5}\right)$ instead of $h_{\text {center }}=O\left(\mathcal{E}^{1 / 6}\right)$, as discussed earlier. The same goes for the conclusion that the integral evaporation rate is either much greater in the pocket than in the neck (scenario III) or of the same order of magnitude (scenario II). It is less evident, however, that the difference concerns in fact the neck scaling, too, but in a more subtle way: While the power laws are the same, for the pocket scenario II there is also a logarithmic modulation involved.

We can also mention at this point that it is a qualitatively different distribution of the local evaporation rate density along the vapor film that is ultimately at the origin of the differences in the asymptotic picture between the present study and Ref. [11]. Indeed, here the local evaporation rate density in the neck is much greater than that in the pocket, so the global evaporation rate is of the same order in the two regions. In contrast, the gas flux density through the substrate considered in Ref. [11] (the counterpart of the evaporation rate density here) is uniform along the gas film underneath the droplet.

Below, consistent with the overall asymptotic analysis underway, we treat the logarithmic terms in a perturbative way. We limit ourselves to a two-term expansion. Namely, we calculate first a leading-order solution to the neck problem in terms of $\ln \delta^{-1}$ and then a corresponding first correction. It is evident that to the leading order here the neck is shaped by the passage of the evaporation flux from the pocket, cf. Eqs. (27) or (28), while evaporation in the neck itself is negligible together with the last term on the left-hand side of Eq. (7). The latter reappear just for the first correction. In this sense, the situation is similar to Ref. [11], with the exception that in this latter study no logarithmic corrections occur but rather just the power-law ones.

\section{J. Two-term logarithmic expansions in the neck}

Inspired by the form of (27) or (28), it is convenient to define $\delta$ by the equation

$$
\frac{12 \mathcal{E} \kappa_{c}}{\delta^{6} \vartheta}\left[f_{\text {aux }}\left(R_{c}\right)+\ln \kappa_{c}-\ln \delta\right] \equiv 1,
$$

where $\mathcal{E} \ll 1$ and $\delta \ll 1$ are of course implied. We shall come back to concrete calculations of $\delta$ later. The smallness parameter $\tilde{\delta}$ for our logarithmic expansions is defined as

$$
\tilde{\delta} \equiv \frac{\vartheta}{f_{\text {aux }}\left(R_{c}\right)+\ln \kappa_{c}-\ln \delta} \ll 1 .
$$

Equation (7) becomes

$$
\frac{d}{d \tilde{x}} \tilde{h}^{3} \frac{d^{3} \tilde{h}}{d \tilde{x}^{3}}-\frac{\tilde{\delta}}{\tilde{h}}=0 .
$$

The condition (28) turns into

$$
\tilde{h}^{3} \frac{d^{3} \tilde{h}}{d \tilde{x}^{3}} \sim-1+\frac{\tilde{\delta}}{\vartheta} \ln \tilde{x} \quad \text { as } \quad \tilde{x} \rightarrow+\infty .
$$

The conditions (6) and (8) do not change. However, we shall rather rewrite (6) in the form (18)-(19) as

$$
\tilde{h} \sim \frac{1}{2} \tilde{x}^{2}+H+O\left(\frac{1}{\tilde{x}^{3}}\right) \quad \text { as } \quad \tilde{x} \rightarrow-\infty,
$$

putting into evidence the choice of the reference along $\tilde{x}$, cf. the discussion below (19).

For what follows, it will also be helpful to rewrite the coordinate expansion (17) in the framework of the 
definitions (29)-(30):

$$
\begin{aligned}
\tilde{h} \sim & \vartheta \tilde{x}+\frac{\tilde{\delta}}{4 \vartheta^{4}} \ln ^{2} \tilde{x}+B \ln \tilde{x}+C \\
& +O\left(\frac{\ln \tilde{x}}{\tilde{x}} ; \tilde{\delta} \frac{\ln ^{2} \tilde{x}}{\tilde{x}} ; \tilde{\delta}^{2} \frac{\ln ^{3} \tilde{x}}{\tilde{x}}\right) \text { as } \tilde{x} \rightarrow+\infty .
\end{aligned}
$$

Likewise, from (20) and (21), we obtain

$$
\begin{aligned}
\tilde{h}^{3} \frac{d^{3} \tilde{h}}{d \tilde{x}^{3}} \sim & \frac{\tilde{\delta}}{\vartheta}\left(\ln \tilde{x}-\frac{3}{2}\right)+2 \vartheta^{3} B \\
& +O\left(\frac{1}{\tilde{x}} ; \tilde{\delta} \frac{\ln \tilde{x}}{\tilde{x}} ; \tilde{\delta}^{2} \frac{\ln ^{2} \tilde{x}}{\tilde{x}}\right) \quad \text { as } \tilde{x} \rightarrow+\infty .
\end{aligned}
$$

Comparing (32) and (35), one finds

$$
B=-\frac{1}{2 \vartheta^{3}}+\frac{3 \tilde{\delta}}{4 \vartheta^{4}}
$$

The solution is sought in the form of an expansion,

$$
\tilde{h}=\tilde{h}_{0}+\tilde{\delta} \tilde{h}_{1}+\cdots,
$$

where, as already mentioned, we limit ourselves here just to two terms. Any other dependent quantity of interest can be represented likewise, viz.,

$$
\vartheta=\vartheta_{0}+\tilde{\delta} \vartheta_{1}+\cdots
$$

and so on.

At the leading order, the last term on the left-hand side of Eq. (31) drops out, and the equation thus can be integrated once, the integration constant being determined from (32) and thus given by the first term on the right-hand side of (32). We thereby obtain the equation

$$
\tilde{h}_{0}^{3} \frac{d^{3} \tilde{h}_{0}}{d \tilde{x}^{3}}=-1
$$

The behavior at infinity is given by (33) and (34) adapted to the leading order:

$$
\tilde{h}_{0} \sim \frac{1}{2} \tilde{x}^{2}+H_{0}+O\left(\frac{1}{\tilde{x}^{3}}\right) \quad \text { as } \quad \tilde{x} \rightarrow-\infty
$$

and

$$
\tilde{h}_{0} \sim \vartheta_{0} \tilde{x}-\frac{1}{2 \vartheta_{0}^{3}} \ln \tilde{x}+C_{0}+O\left(\frac{\ln \tilde{x}}{\tilde{x}}\right) \quad \text { as } \tilde{x} \rightarrow+\infty,
$$

where it has been taken into account that

$$
B_{0}=-\frac{1}{2 \vartheta_{0}^{3}}
$$

in accordance with (36).

The formulated boundary-value problem, of which $\vartheta_{0}, C_{0}$, and $H_{0}$ are nonlinear eigenvalues, is solved numerically. The problem is parameter free, so its solution is obtained once and for all. We recur to the shooting method and proceed from $+\infty$ to $-\infty$. As it has already been discussed in an earlier context, the value of $\vartheta_{0}$ is sought to obtain the right coefficient at $O\left(\tilde{x}^{2}\right)$ in (40). The value of $C_{0}$, which amounts in (41) to a mere shift along $\tilde{x}$, then can be adjusted to have the right coefficient (here zero) also at $O\left(\tilde{x}^{1}\right)$ in (40). The value of $H_{0}$ is thereby obtained simply as a result of the solution.
Similarly, the problem for the first correction is reduced to

$$
\begin{gathered}
\frac{d}{d \tilde{x}}\left(\tilde{h}_{0}^{3} \frac{d^{3} \tilde{h}_{1}}{d \tilde{x}^{3}}-3 \frac{\tilde{h}_{1}}{\tilde{h}_{0}}\right)-\frac{1}{\tilde{h}_{0}}=0, \\
\tilde{h}_{1} \sim H_{1}+O\left(\frac{1}{\tilde{x}^{3}}\right) \text { as } \tilde{x} \rightarrow-\infty, \\
\tilde{h}_{1} \sim \vartheta_{1} \tilde{x}+\frac{1}{4 \vartheta_{0}^{4}} \ln ^{2} \tilde{x}+B_{1} \ln \tilde{x}+C_{1}+O\left(\frac{\ln ^{2} \tilde{x}}{\tilde{x}}\right) \\
\quad \text { as } \tilde{x} \rightarrow+\infty, \\
B_{1}=\frac{3}{2 \vartheta_{0}^{4}} \vartheta_{1}+\frac{3}{4 \vartheta_{0}^{4}},
\end{gathered}
$$

where the leading-order solution is already known. As the problem is linear, the task reduces to finding the appropriate linear combination of various numerically obtained solutions: a particular solution of the inhomogeneous problem and linearly independent solutions of the homogeneous problem. This leads, of course, to a simultaneous determination of $\vartheta_{1}$, $C_{1}$, and $H_{1}$. Just as for the leading order, the problem for the first correction is parameter free and its solution is obtained once and for all.

On the contrary, the values of $\delta$ and $\tilde{\delta}$, defined in (29) and (30), to which we are back now, are here to be calculated on a case-by-case basis, i.e., for each particular value of $\mathcal{E}$ and of the size of the droplet [earlier followed by means of $R_{\text {max }}$, the quantities $R_{c}$ and $\kappa_{c}$ being functions of it as for the equilibrium shape of a superhydrophobic sessile droplet, see Fig. 7(a)]. Using the numerically obtained ("universal") values

$$
\vartheta_{0}=0.963, \quad \vartheta_{1}=-0.672
$$

in the two-term expansion (38), what we actually do is to solve the system of equations (29), (30), and (38) for the three unknowns $\delta, \tilde{\delta}$, and $\vartheta$. Next, we can use this in two-term expansions like (37), together with the universal numerically computed results for the leading order and the first correction, to obtain concrete predictions. These can be rendered in terms of the original dimensionless variables, used in Sec. II, by means of (4) and (5). In particular, $h_{\text {neck }}$ will be calculated here by numerically minimizing the two-term expansion (37) to yield $\tilde{h}_{\text {neck }}$ and subsequently using (5) to remove the tilde. Having computed $\delta$ and $\vartheta$, we are now in a position to make concrete predictions for the pocket, too, using (12) and (10). In particular, evaluation at $r=0$ yields

$$
h_{\text {center }}=\delta \vartheta \frac{J_{0}(0)-J_{0}\left(R_{c}\right)}{J_{1}\left(R_{c}\right)} .
$$

Certain results of a parametric study carried out in this way are presented in the following subsection.

\section{K. Results and discussion of MAE}

The results of a parametric study versus the droplet size are shown in Figs. 7(b)-7(d). They correspond to $\mathcal{E}=1.21 \times$ $10^{-6}$, one of the examples considered in Sec. III. The results of the present asymptotic analysis are depicted by dashed (green) lines in all figures. The solid (blue) lines stand for the computation undertaken in Sec. III, reproduced here for the sake of comparison. The dot-dashed (red) lines represent 
(a)

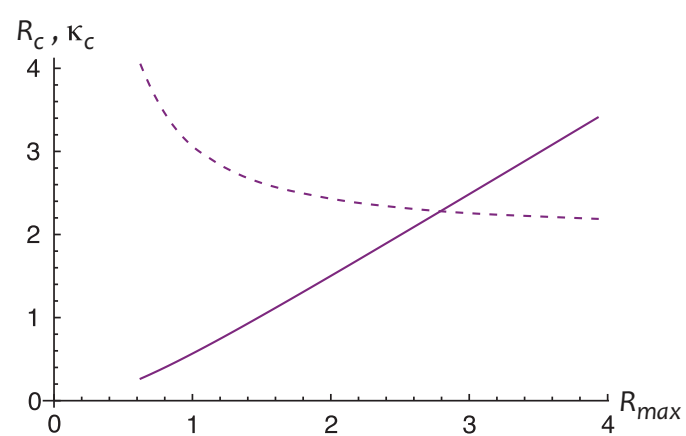

(c)

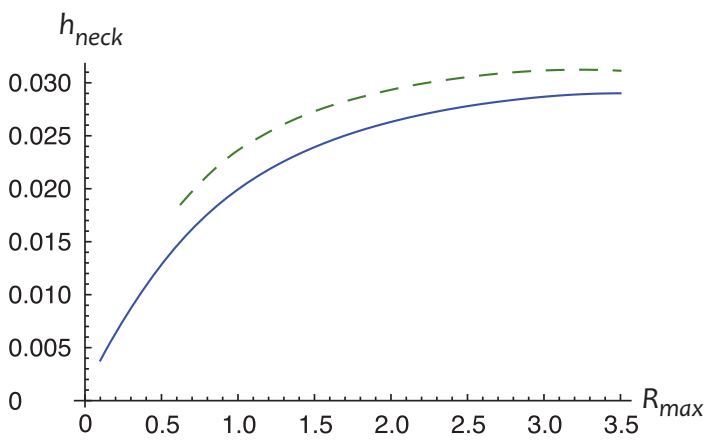

(b)

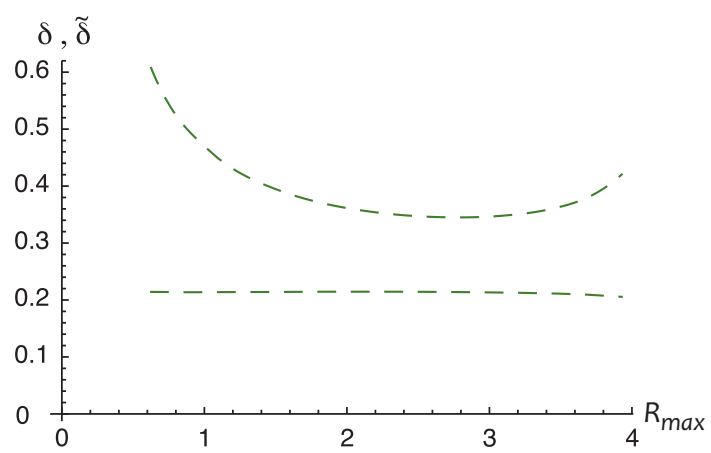

(d)

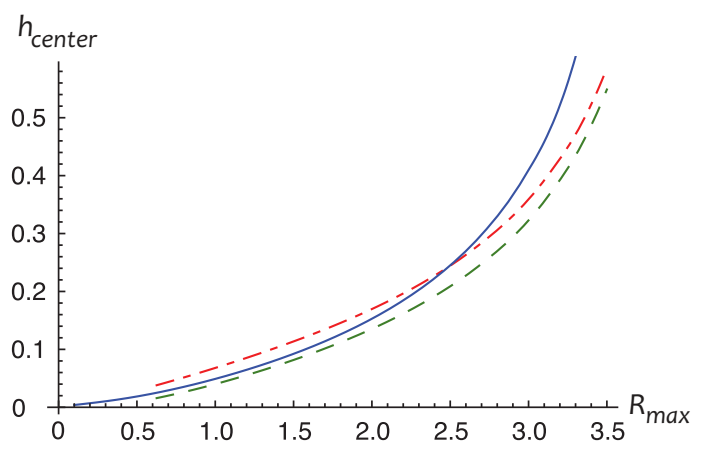

FIG. 7. (Color online) Various quantities as functions of the maximum horizontal radius of the drop for $\mathcal{E}=1.21 \times 10^{-6}$ (water at $T_{w}^{*}=370^{\circ} \mathrm{C}$ ) when relevant. (a) Contact radius $R_{c}$ (solid line) and curvature at the contact line $\kappa_{c}$ (dotted lined) of the associated equilibrium superhydrophobic droplet. (b) Smallness parameters of the problem ( $\delta$ the lower curve and $\tilde{\delta}$ the upper curve). (c) $h_{\text {neck }}$ results of the present asymptotic analysis (dashed, green line) and of the computation in Sec. III (solid blue line). (d) $h_{\text {center }}$, with the line formats meaning the same as above, while the dot-dashed (red) line is for the semiheuristic result accounting for droplet elevation.

a semiheuristic modification (see below) applied to the results of the asymptotic analysis.

As shown in Fig. 7(b), the smallness parameters $\delta$ and $\tilde{\delta}$ used in the asymptotic expansions are actually not very small in practice. Nonetheless, the asymptotic results for the neck thickness $h_{\text {neck }}$ agree rather well with the numerical ones obtained in Sec. III. The agreement appears to be less spectacular, though, for $h_{\text {center }}$, the maximum height of the vapor pocket (attained at the symmetry axis), although the tendency is still well followed. We note that for sufficiently small $R_{\max }$, when the calculated values of $h_{\text {neck }}$ and $h_{\text {center }}$ become close to one another, the asymptotic analysis is deemed to lose its meaning, and the parametric study is not continued into this domain. The semiheuristic result (which does not pertain to a rigorous asymptotic analysis) also shown in Fig. 7(d) is obtained by formally adding to the vapor pocket thickness the elevation calculated for the upper, equilibrium part of the Leidenfrost droplet. In terms of the asymptotic analysis, this elevation corresponds to $\left(H_{0}+\tilde{\delta} H_{1}\right) \delta^{2} / \kappa_{c}$ [cf. the paragraph following Eq. (19) and the scaling (5)]. In this way, it proves possible to encompass the solid (blue) curve, albeit for not-too-large $R_{\max }$ values. One of the reasons for a worsening agreement at large $R_{\max }$ could be related to a thickening of the vapor pocket as $R_{c}$ approaches 3.83 and a consequent deterioration of the thin-film approximation relied on here (recall that it is an exact expression for the curvature that is used in the formulation of Sec. II).

Shown in Figs. 8(a)-8(d) are the same quantities $\delta, \tilde{\delta}, h_{\text {neck }}$, and $h_{\text {center }}$ but now as functions of $\mathcal{E}$ for $R_{\max }=3$ and $R_{\max }=$
1, similarly to a representation used in Fig. 5. In the presented range, the slope of the curves in Fig. 8(a) varies within $0.158 \pm 0.001$ for $R_{\max }=3$ and $0.156 \pm 0.002$ for $R_{\max }=1$ (with larger values to the left). In view of Eqs. (29) and (30), its being rather close to $1 / 6$ (the value that would be obtained from "naive" considerations, see the beginning of this section) must be due to a relatively weak dependence of $\tilde{\delta}$ on $\mathcal{E}$, as witnessed by Fig. 8(b). It is physically more remarkable for us, however, that a similar nearly constant-slope behavior is with a high precision observed in Fig. 8 (c) for $h_{\text {neck }}: 0.321 \pm 0.002 \approx 1 / 3$ for $R_{\max }=3$ and $0.318 \pm 0.002 \approx 1 / 3$ for $R_{\max }=1$, and hence $h_{\text {neck }} \approx O\left(\mathcal{E}^{1 / 3}\right)$. Thus, no logarithmic modulation is really felt when applied to practical values of $\mathcal{E}$ (and a few orders of magnitude lower), even in the framework of the results of the asymptotic analysis. This goes along nicely with the observation of this same asymptotics-determined power law in the modeling results presented in Sec. IV. In contrast, as far as $h_{\text {center }}$ is concerned [Fig. 8(d)], the power-law exponent proves to be less stable along the curves and also differs markedly for the two versions (asymptotically rigorous and semiheuristic) used. A most representative picture is perhaps given by averaging in each case between the dashed (green) and dot-dashed (red) curves. One thereby obtains $0.152 \pm 0.005$ for $R_{\max }=3$ and $0.16 \pm 0.01$ for $R_{\max }=1$. While this is still close to the expected asymptotics-determined (without logarithmic modification) exponent $1 / 6$, the precision of that for $h_{\text {center }}$ is lower than earlier for $h_{\text {neck }}$. At the same time, there is a clear tendency for an increase of the exponent at smaller $R_{\max }$, manifest in the semiheuristic variant. Somehow, 
(a)

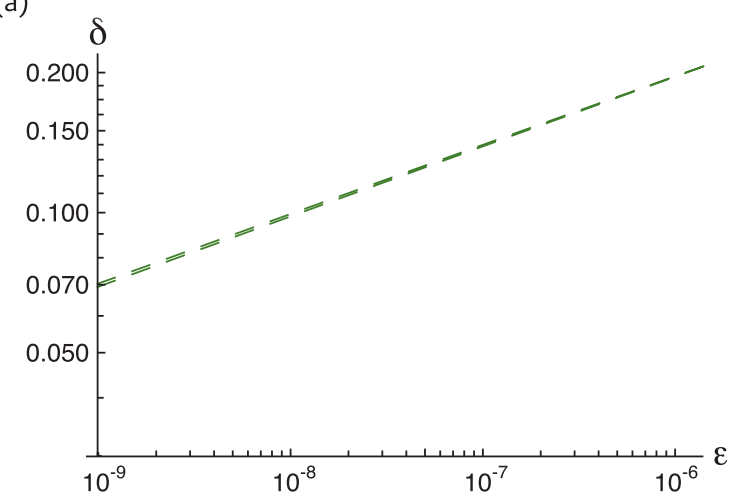

(c)

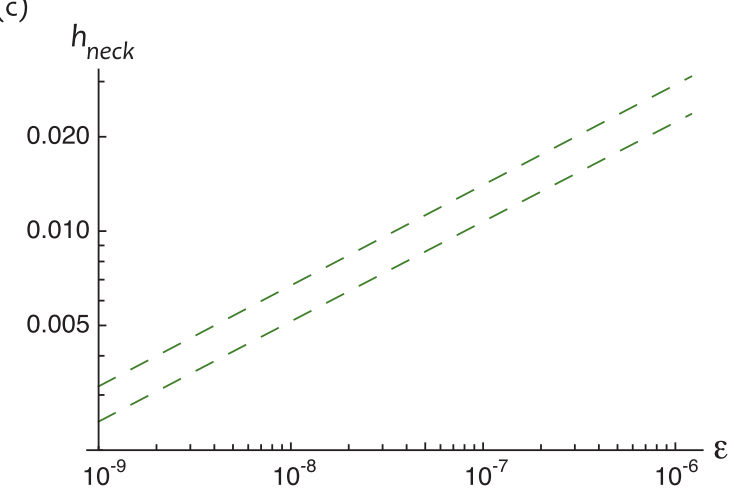

(b)

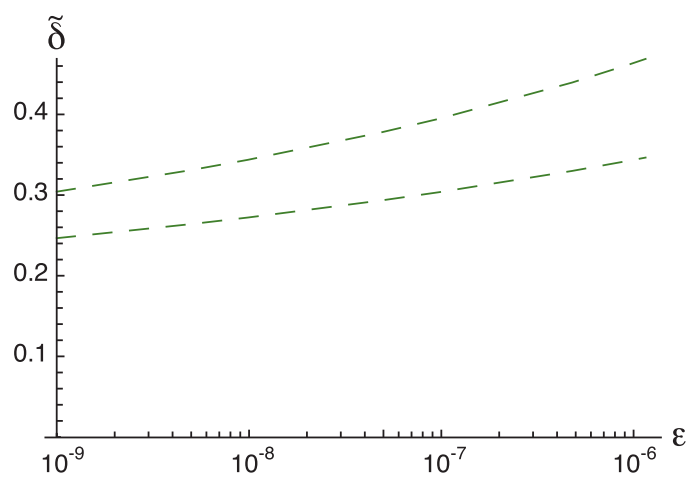

(d)

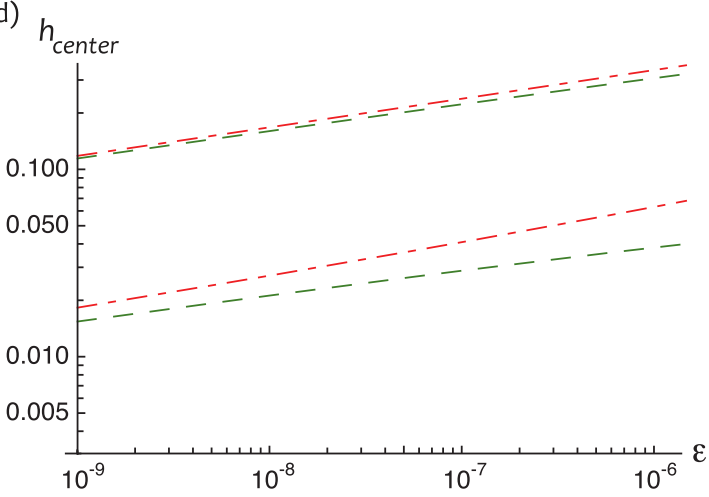

FIG. 8. (Color online) Various quantities as functions of $\mathcal{E}$ for $R_{\max }=3$ [lower curves in (a) and (b) and upper ones in (c) and (d)] and $R_{\max }=1$ (vice versa). The curves nearly coincide in (a). The line format meaning is the same as described in the caption to Fig. 7.

this also conforms to the modeling results provided in Sec. IV: While an exponent equal to $1 / 6$ is still recovered at $R_{\max }=3$ and $R_{\max }=1$, it loses its asymptotics-determined value and becomes higher for smaller $R_{\max }$.

\section{Closing remarks to the MAE analysis}

The neck-pocket structure of the vapor film underneath sufficiently large Leidenfrost droplets has been confirmed by means of matched asymptotic expansions in the limit $\mathcal{E} \rightarrow 0$. Various pocket scenarios have been explored, among which the one corresponding, to leading order, to an equilibrium trapped bubble shape with an apparent contact angle is shown to be appropriate here and to correspond to the modeling results of Sec. III. The asymptotic results are found to compare reasonably well with that modeling, although their quality must clearly deteriorate for smaller $R_{\max }$, when $h_{\text {neck }}$ becomes comparable with $h_{\text {center }}$ for a given $\mathcal{E}$. The scalings $h_{\text {neck }}=O\left(\mathcal{E}^{1 / 3}\right)$ and $h_{\text {center }}=O\left(\mathcal{E}^{1 / 6}\right)$ established in Sec. IV are confirmed to be asymptotics determined, yet with a twist that they must in fact be logarithmically modulated in the true asymptotic limit (and the asymptotic expansion must actually be carried out in terms of the logarithm of the smallness parameter). Furthermore, the same is true for the scaling $\mathcal{E}^{5 / 6}$ of the the global evaporation rates in the pocket and in the neck. No such logarithmic modulations are observed for practically small values of $\mathcal{E}$, though. At the same time, we have noticed that the scaling $h_{\text {center }}=O\left(\mathcal{E}^{1 / 6}\right)$ seems to be less persistent at smaller $R_{\text {max }}$ than $h_{\text {neck }}=O\left(\mathcal{E}^{1 / 3}\right)$. This is apparently why, as shown in Sec. IV, the power-law exponent is more prone, for $h_{\text {center }}$ than for $h_{\text {neck }}$, to deviate from its asymptotics-determined value as $R_{\max }$ is decreased.

\section{CONCLUDING REMARKS}

In summary, the rather "minimalist" model proposed here covers the full range of stable Leidenfrost drops (limited by the take-off and by the chimney formation) and appears to be in excellent agreement with recent measurements of vapor pocket and neck characteristics by Burton et al. [9]. A less satisfactory, yet reasonable, agreement is found with earlier results of Biance et al. [10], whose scaling analysis is here refined by accounting for the actual geometry of the vapor film underneath the drop. Furthermore, a MAE analysis reveals a logarithmic modulation of the scaling (power) laws in the asymptotic limit, although this is not observed here for practical values of the smallness parameter. Puzzling enough is the fact that no threshold is predicted, i.e., droplets levitate however small the superheat, at odds with experimental evidence. However, the present model can be rather easily generalized by additional effects such as substrate roughness, for instance, which could help solving this crucial issue. We also hope that this new theoretical framework will stimulate further progress, e.g., in the context of drop manipulation by ratchets or external fields.

\section{ACKNOWLEDGMENTS}

The authors gratefully acknowledge financial support of Fonds de la Recherche Scientifique-FNRS 
(FRFC-ODILE contract, Research Associate Position of SD and Senior Research Associate Position of PC), ESABELSPO (PRODEX projects), and BELSPO (IAP 7/38
$\mu$-MAST project). This research was also carried out under COST Action MP1106's umbrella.
[1] J. G. Leidenfrost, De Aquae Communis Nonnullis Qualitibus Tractatus, Part 2 (Hermannus Ovenius, Duisburg, 1756).

[2] D. Quéré, Annu. Rev. Fluid Mech. 45, 197 (2013).

[3] H. Linke, B. J. Alemán, L. D. Melling, M. J. Taormina, M. J. Francis, C. C. Dow-Hygelund, V. Narayanan, R. P. Taylor, and A. Stout, Phys. Rev. Lett. 96, 154502 (2006).

[4] G. Dupeux, M. Le Merrer, C. Clanet, and D. Quéré, Phys. Rev. Lett. 107, 114503 (2011).

[5] T. R. Cousins, R. E. Goldstein, J. W. Jaworski, and A. I. Pesci, J. Fluid Mech. 696, 215 (2012).

[6] Á. G. Marín, D. A. del Cerro, G. R. B. E. Römer, B. Pathiraj, A. Huis In't Veld, and D. Lohse, Phys. Fluids 24, 122001 (2012).

[7] F. Celestini and G. Kirstetter, Soft Matter 8, 5992 (2012).

[8] K. Piroird, C. Clanet, and D. Quéré, Phys. Rev. E 85, 056311 (2012).
[9] J. C. Burton, A. L. Sharpe, R. C. A. van der Veen, A. Franco, and S. R. Nagel, Phys. Rev. Lett. 109, 074301 (2012).

[10] A.-L. Biance, C. Clanet, and D. Quéré, Phys. Fluids 15, 1632 (2003).

[11] J. H. Snoeijer, P. Brunet, and J. Eggers, Phys. Rev. E 79, 036307 (2009).

[12] P. Brunet and J. H. Snoeijer, Eur. Phys. J. Spec. Top. 192, 207 (2011).

[13] F. Celestini, T. Frisch, and Y. Pomeau, Phys. Rev. Lett. 109, 034501 (2012).

[14] Y. Pomeau, M. Le Berre, F. Celestini, and T. Frisch, C. R. Mecanique 340, 867 (2012).

[15] L. Mahadevan and Y. Pomeau, Phys. Fluids 11, 2449 (1999).

[16] Note that Biance et al. [10] predict this exponent only for $R_{\max } \ll$ 1 , while an exponent $1 / 4$ is found for $R_{\max } \gg 1$. Pomeau et al. [14] find the right exponent $1 / 3$ in both asymptotic cases. 